\title{
Cervical Spinal Surgery of a Patient with Hemophilia and Ehlers-Danlos Syndrome: Anesthetic Considerations
}

\author{
Soghomonyan $\mathrm{S}^{1 *}$, Khan $\mathrm{S}^{2}$, Li $\mathrm{L}^{1}$, Soghomonyan \\ $\mathbf{G}^{3}$ and Grossbach $\mathbf{A J}^{4}$ \\ ${ }^{1}$ Ohio State University Wexner Medical Center, \\ Department of Anesthesiology, Columbus, OH, USA \\ ${ }^{2}$ Ohio State University Wexner Medical Center, \\ Department of Orthopedic Surgery, Columbus, OH, USA \\ ${ }^{3}$ Visiting student, Claremont McKenna College, \\ Claremont, CA, USA \\ ${ }^{4}$ Ohio State University Wexner Medical Center, \\ Department of Neurological Surgery, Columbus, OH, USA \\ *Correspondling author: Soghomonyan S, Department \\ of Anesthesiology, Ohio State University Wexner \\ Medical Center, N411 Doan Hall, 410 West 10th Avenue, \\ Columbus, OH 43210, USA
}

Received: September 29, 2021; Accepted: October 21, 2021; Published: October 28, 2021

\section{Abbreviations}

EDS: Ehlers-Danlos Syndrome; i.v: intravenous; U/kg: Units/ kilogram; mg: Milligram(s); mcg/kg/min/: Micrograms/kilogram/ minute; MAC: Minimum Alveolar Concentration; ml: Milliliter; ddAVP: 1-deamino-8-D-Arginine Vasopressin; ICU: Intensive Care Unit

\section{Case Presentation}

Major spinal procedures are frequently associated with an increased risk of significant intraoperative blood loss, sometimes, exceeding 5 liters, which requires blood transfusion (in 50-81\% of procedures) [1]. The risk of bleeding substantially increases in patients with concomitant hemostatic disorders. We present a case of successful surgery in a patient with cervical spinal tumor (schwannoma), hemophilia A, and Ehlers-Danlos Syndrome (EDS). The patient consented for this publication.

A 32-years-old male patient (76.2kg, body mass index 27.1) was admitted with complains of left shoulder pain and arm numbness. Objective examination revealed radicular symptoms on the left, and magnetic resonance imaging demonstrated a $2.4 \mathrm{~cm}$ cystic, intradural, extramedullary lesion within the left ventral aspect of the spinal canal with compression and flattening of the spinal cord and extension into the left C5 and C6 root foramina (Figure 1). Based on the findings, a leading diagnosis of cystic schwannoma was made.

The patient's medical history was significant for arterial hypertension, well-controlled hypothyroidism, hemophilia A, and EDS - hypermobile variant. According to his past medical history, the patient had developed a spontaneous intracranial hemorrhage as an infant requiring neonatal intensive care unit admission.

The patient had severe hemophilia A with factor VIII $<1 \%$, and his coagulation was managed with periodic injections of Nurwiq - a recombinant anti-hemophilic factor (Octapharma, Paramus, NJ).
There was no previous history of vascular fragility or internal organ ruptures related to his EDS.

The patient was scheduled for surgical resection of the tumor, and during the whole perioperative period, his coagulation status was managed by the hematology service.

He received 1 dose of Nuwiq - 50U/kg, on the day before surgery, and the treatment was continued with a dose of $35 \mathrm{U} / \mathrm{kg}$ every 12 hours for the subsequent 48 hours. After discharge, the patient continued receiving daily Nurwiq (4,350 units) for 9 days.

\section{Anesthetic management}

After establishing an i.v. access, the patient was premedicated with $2 \mathrm{mg}$ of midazolam before his transfer to the operating room. Following preoxygenation, anesthesia was induced with standard doses of fentanyl, lidocaine, and propofol. The muscle relaxation was achieved with administration of $50 \mathrm{mg}$ rocuronium, which was not redosed to allow for monitoring of the evoked motor responses during manipulations in proximity to the spinal cord.

The patient was intubated with a lubricated \# 6 cuffed endotracheal tube. An additional i.v. catheter was placed, and infusions of remifentanil, $0.05-0.08 \mathrm{mcg} / \mathrm{kg} / \mathrm{minute}$, propofol, $50 \mathrm{mcg} / \mathrm{kg} / \mathrm{minute}$, were started along with inhalation of $0.5 \mathrm{MAC}$ of sevoflurane in 1:1 air - oxygen mixture. The Blood Pressure (BP) was supported with infusion of phenylephrine titrated to maintain mean $\mathrm{BP}>65 \mathrm{mmHg}$. The right radial artery was cannulated with ultrasound guidance to monitor the BP throughout surgery. Intraoperatively, the motor and somatosensory evoked potentials were registered. The total duration of anesthesia was 6 hours.

The intraoperative blood loss was insignificant (150ml) and did not require any transfusions. The patient remained hemodynamically stable throughout surgery. He received a total of $2800 \mathrm{ml}$ of crystalloids, and the urinary output was $850 \mathrm{ml}$. Intraoperative antibacterial prophylaxis was achieved with cefazolin, and dexamethasone with
Austin J Anesthesia and Analgesia - Volume 9 Issue 2 - 2021 ISSN : 2381-893X | www.austinpublishing group.com Soghomonyan et al. (C) All rights are reserved
Citation: Soghomonyan S, Khan S, Li L, Soghomonyan G and Grossbach AJ. Cervical Spinal Surgery of a Patient with Hemophilia and Ehlers-Danlos Syndrome: Anesthetic Considerations. Austin J Anesthesia and Analgesia. 2021; 9(2): 1101. 


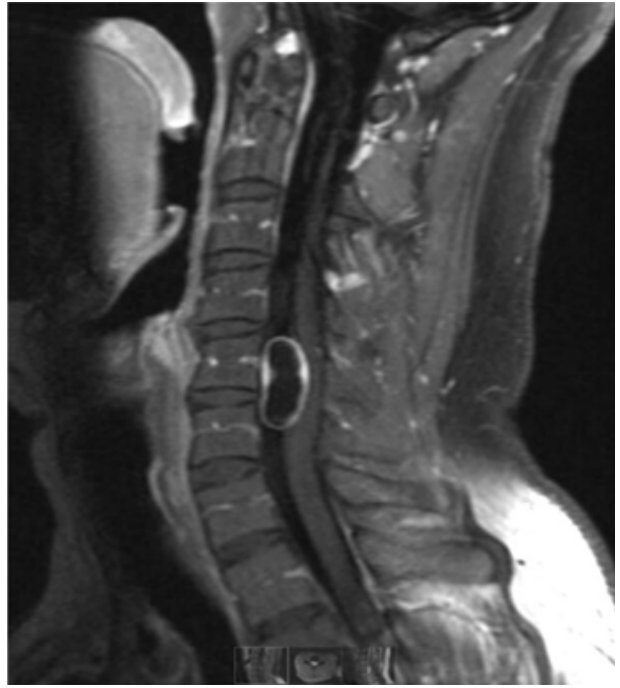

Figure 1: Magnetic resonance imaging demonstrating the tumor growing over the left ventral aspect of the cervical spinal cord causing compression.

ondansetron were administered for prophylaxis of postoperative nausea and vomiting. Pressure-controlled and volume guaranteed ventilatory mode was used for respiratory support avoiding high intrapulmonary pressures: the peak pressure remained $\leq 18 \mathrm{~cm} \mathrm{H}_{2} \mathrm{O}$ throughout anesthesia.

\section{Surgery}

Given the anterior location of the tumor, a standard posterior or posterolateral approach was not ideal so an anterior C5 corpectomy with C4-5 and C5-6 discectomies was performed. The tumor was approached and resected using standard intradural microsurgical techniques under fluoroscopic and ultrasound imaging guidance. A biomechanical interbody cage was placed at C5, and a C4-6 anterior cervical fusion was performed. The wound layers were closed and sterile dressing was applied to the incision site. The patient was extubated upon completion of the procedure and was transferred to the neurosurgical ICU for further postoperative care and monitoring.

The postoperative course was uncomplicated, and the patient was discharged home to continue the anti-hemophilia treatment under the supervision of his hematologist. In the postoperative period, no bleeding or neurological deterioration were observed.

\section{Discussion}

Clotting disorders predispose to major bleeding during surgery. Patients with hemophilia are considered a group of the highest risk, and surgical planning for this category of patients requires a multidisciplinary approach and proper preparation to avoid surgical disasters.

Hemophilia $\mathrm{A}$ is an X-linked disease with an incidence of approximately $1 / 5000$ male live births which is characterized by defective or deficient clotting factor VIII [2].

There is a limited number of reports on anesthetic management of patients with hemophilia undergoing neurosurgical procedures [35]. Aouba A et al. (2010) described a patient with hemophilia, who underwent successful removal of a temporo-parietal glioblastoma [3].
The patient produced anti-factor VIII autoantibodies, so recombinant activated factor VII was administered in the perioperative period, which directly activates factor $\mathrm{X}$ bypassing the steps requiring factor VIII or factor IX (seen in hemophilia A and B, respectively).

Recombinant activated factor VII is sometimes used off-label to reverse anticoagulation, prevent progression of intracranial hemorrhage, and reduce the intraoperative bleeding [6]. However, its benefits must be weighed against the risk of thrombotic complications.

Prior to surgery, our patient's factor VIII deficit was effectively compensated with Rurwiq, so a decision was made to maintain the patient on the same drug with dose adjustments to achieve factor VIII level of $100 \pm 10 \%$ during and after surgery.

Another drug temporarily restoring the factor VIII level used during minor procedures in patients with hemophilia is 1-deamino8-D-arginine vasopressin (ddAVP). However, major procedures, as in our case, require full factor replacement to avoid significant intraoperative bleeding.

In addition to factor replacement therapy, Kobayashi K. and coauthors (2018) recommend using additional measures to reduce the intraoperative blood loss in hemophilic patients undergoing spinal surgery: controlled hypotension, cotton fibrillary collagen applied to reach hemostasis, and intraoperative blood salvage [5].

Another factor increasing the risk of bleeding in our patient was the history of EDS - a rare genetic disorder of collagen synthesis with all three major inheritance patterns (autosomal dominant, autosomal recessive and X-linked) described in association with it [7]. The overall incidence is $1 / 10,000-1 / 25,000$ with no ethnic predisposition [8].

There are 13 subtypes of the disease, and the main ones are hypermobile, vascular, kyphoscoliotic, arthrochalasia, and dermatosparaxis. Among them, the vascular type carries the highest potential for severe morbidity and mortality related to vascular and visceral tissue fragility [8-10].

Even though laboratory tests for EDS are not prognostic for evaluation of the bleeding risk, platelet aggregation abnormalities are seen in about $26 \%$ of patients [8]. The platelet dysfunction is explained by presence of defective collagen in the vessels, which leads not only to weakness of the vascular wall but also to altered interaction with platelets and von Willebrand factor thus interfering with the primary thrombus formation [11].

Factors that require anesthesiologist's attention in patients with EDS are: fragile and poorly healing skin, excessive bleeding, predisposition to spontaneous pneumothorax, especially with high ventilatory pressures and excessive tidal volumes, easy joint dislocation, cardiac mitral valve prolapse, presence of coronary and cerebral aneurysms, spontaneous dissections or ruptures of major vessels, small mouth and microretrognathia, mucosal damage and bleeding as a result of endotracheal intubation $[7,12]$.

We took precautions to minimize the perioperative risks for our patient. The coagulation deficiency was corrected based on the hematologist's recommendations with the aim to fully replace the deficient factor VIII. Pressure points were carefully padded to reduce skin damage related to skin fragility observed in patients with EDS. 
We used a small lubricated endotracheal tube, which was placed with extreme care to avoid mucosal damage. Intraoperatively, high ventilatory pressures and inspiratory volumes were avoided to decrease the risk of pneumothorax seen in some patients with EDS.

Wiesmann T, et al. (2014) recommend avoiding central venous catheterizations and arterial cannulations in patient with EDS with a history of vascular fragility. [8] In our case, invasive arterial pressure monitoring was required, so an arterial cannula was inserted after induction and intubation with great caution and under ultrasound guidance to minimize vascular trauma. We also took into consideration the type of HDS in our patient (hypermobile type) and no previous evidence of vascular fragility in his past history.

Special attention was paid to meticulous hemostasis. No significant bleeding or other problems were encountered during surgery. The tumor was successfully removed and surgery was completed with anterior cervical fusion.

The postoperative course was uneventful. The patient's neurological symptoms resolved and he was discharged home.

\section{Conclusion}

Our patient had two rare genetic disorders, which placed him at risk for perioperative bleeding. The multi-disciplinary approach and proper planning of anesthesia and surgery allowed for safe and uneventful completion of the procedure. Recognition of all risk factors related to the patient's hemophilia A and EDS, and application of above discussed preventive measures helped to successfully conduct the surgical procedure and avoid serious complications.

\section{References}

1. Rankin D, Zuleta-Alarcon A, Soghomonyan S, Abdel-Rasoul M, CastellonLarios K, Bergese SD. Massive blood loss in elective spinal and orthopedic surgery: Retrospective review of intraoperative transfusion strategy. J Clin Anesth. 2017; 37: 69-73.
2. Mistry T, Dogra N, Chauhan K, Shahani J. Perioperative Considerations in a Patient with Hemophilia A: A Case Report and Review of Literature. Anesth Essays Res. 2017; 11: 243-245.

3. Aouba A, Dezamis E, Sermet A, Rothschild C, Hermine O, Lasne D, Torchet MF. Uncomplicated neurosurgical resection of a malignant glioneuronal tumour under haemostatic cover of rFVIIa in a severe haemophilia patient with a high-titre inhibitor: a case report and literature review of rFVIla use in major surgeries. Haemophilia. 2010; 16: 54-60.

4. Palsma R, Gravbrot N, Bina R, Agastya M, Kasoff WS. Deep Brain Stimulation Surgery for Essential Tremor in a Patient with Type A Hemophilia. World Neurosurg. 2020; 139: 158-162.

5. Kobayashi K, Imagama S, Ando K, Ito K, Tsushima M, Morozumi M, et al. Perioperative Management of Patients with Hemophilia during Spinal Surgery. Asian Spine J. 2018; 12: 442-445

6. Zhou JJ, Chen T, Nakaji P. Intraoperative Blood and Coagulation Factor Replacement during Neurosurgery. Neurosurg Clin N Am. 2018; 29: 547-555.

7. Kuczkowski KM, Benumof JL. Cesarean section and Ehlers-Danlos syndrome: choice of anesthesia. Int J Obstet Anesth. 2002; 11: 222-224.

8. Wiesmann T, Castori M, Malfait F, Wulf H. Recommendations for anesthesia and perioperative management in patients with Ehlers-Danlos syndrome(s). Orphanet J Rare Dis. 2014; 9: 109.

9. Ohshita N, Kanazumi M, Tsuji K, Yoshida H, Morita S, Momota Y, et al. Anesthetic Management of a Patient With Ehlers-Danlos Syndrome. Anesth Prog. 2016; 63: 204-207.

10. Artoni A, Bassotti A, Abbattista M, Marinelli B, Lecchi A, Gianniello F, et al. Hemostatic abnormalities in patients with Ehlers-Danlos syndrome. J Thromb Haemost. 2018; 16: 2425-2431.

11. Jesudas R, Chaudhury A, Laukaitis CM. An update on the new classification of Ehlers-Danlos syndrome and review of the causes of bleeding in this population. Haemophilia. 2019; 25: 558-566.

12. Wakabayashi R, Tanaka S, Tsuchiyama K, Yamamoto K, Maruyama Y, Numata K, et al. Anesthetic management of a patient with musculocontractural Ehlers-Danlos syndrome undergoing scoliosis surgery. JA Clin Rep. 2020; 6: 46. 